\title{
Aa. Vv. Arrigo Beyle «Romano» (1831-1841). Stendhal fra Storia, Cronaca, Letteratura, Arte, atti del convegno internazionale, Roma, 24-26 ottobre 2002
}

\section{Annalisa Bottacin}

\section{(2) OpenEdition}

\section{Journals}

\section{Edizione digitale}

URL: http://journals.openedition.org/studifrancesi/27723

DOI: $10.4000 /$ studifrancesi.27723

ISSN: 2421-5856

\section{Editore}

Rosenberg \& Sellier

\section{Edizione cartacea}

Data di pubblicazione: 31 décembre 2006

Paginazione: 616-618

ISSN: 0039-2944

\section{Notizia bibliografica digitale}

Annalisa Bottacin, «Aa. Vv. Arrigo Beyle «Romano» (1837-1847). Stendhal fra Storia, Cronaca, Letteratura, Arte, atti del convegno internazionale, Roma, 24-26 ottobre 2002 », Studi Francesi [Online], 150 (L | III) | 2006, online dal 30 novembre 2015, consultato il 08 novembre 2020. URL : http://

journals.openedition.org/studifrancesi/27723; DOI : https://doi.org/10.4000/studifrancesi.27723

Questo documento è stato generato automaticamente il 8 novembre 2020 .

\section{cc) $(8)$}

Studi Francesi è distribuita con Licenza Creative Commons Attribuzione - Non commerciale - Non opere derivate 4.0 Internazionale. 


\title{
Aa. Vv. Arrigo Beyle «Romano» (1831-1841). Stendhal fra Storia, Cronaca, Letteratura, Arte, atti del convegno internazionale, Roma, 24-26 ottobre 2002
}

\author{
Annalisa Bottacin
}

\section{NOTIZIA}

AA. VV. Arrigo Beyle «Romano» (1831-1841). Stendhal fra Storia, Cronaca, Letteratura, Arte, atti del convegno internazionale, Roma, 24-26 ottobre 2002, a cura di M. COLESANTI, H. DE JACQUELOT, L. NORCI CAGIANO, A.M. SCAIOLA, Roma, Edizioni di Storia e Letteratura, 2004, «Quaderni di Cultura Francese a cura della Fondazione Primoli», n 38, pp. 324, ill.

1 Nella storia degli studi romani dedicati a Stendhal, questo volume ricostruisce, analizzando vari aspetti della figura e dell'opera di Henri Beyle in quegli anni console di Francia a Civitavecchia, un vissuto ancora in parte da rivisitare, e giunge a conclusioni di alto rigore scientifico che si prefiggono di proporre, ben riuscendovi, un'attenzione particolare sul lungo passaggio nello Stato della Chiesa dello scrittore grenoblese.

Nella Premessa, Massimo colESANTI visualizza subito la novità dei contributi, sottolineando che «[t]utte le comunicazioni hanno rilevato testimonianze o documenti inediti, o mostrato e analizzato immagini ed episodi notissimi, ma collocati in una luce nuova, in un contesto interdisciplinare e diverso dai tanti luoghi comuni finora ripetuti» (p. 7). Ed è ancora Colesanti, nella sua bella e corposa prolusione dal titolo, «La noia "feconda" di Stendhal a Roma» (pp. 1-10), a porsi una domanda affatto retorica, affrontando un problema fondamentale nella vita romana di Stendhal: l'eminente stendhaliano nota infatti che «si è esagerato non poco, crediamo, sulla noia che 
Stendhal avverte a Roma, sui suoi continui sfoghi di malumore, sulle sue proteste e recriminazioni, che leggiamo soprattutto nelle sue lettere agli amici e alle amiche di Parigi e che hanno a volte il tono di una posa salottiera e vittimistica» (p. 3), in quanto lo scrittore par ben essersi integrato nella quotidianità romana, dove trovava ciò che più gli mancava a Civitavecchia, cioè la conversazione e la frequentazione dei salotti. «[A] Roma, spesso, - prosegue Colesanti - molto spesso, egli non mena certo una vita triste, torpida, indolente, ma fervida di relazioni, di affetti, di impegni, di visite culturali, e di intenso lavoro creativo» (p. 4). Soltanto dai titoli delle tre sezioni, che il volume presenta, si evidenzia quanto sia vasto e onnicomprensivo questo regesto composto da altissimi nomi di critici e studiosi.

3 Per venire nel dettaglio, la prima sezione si occupa di Henri Beyle console di Francia a Civitavecchia. I primi due studi si distinguono per l'integrazioni nei testi di nuovi apporti inediti: Elaine WILLIAMSON (pp. 21-37), già autrice di un fondamentale lavoro su Stendhal e l'olanda, si pone davanti alla questione della figura del console Beyle, in un attento esame dei suoi rapporti non sempre facili con le autorità francesi quanto con quelle romane, sottoponendo al lettore una raffinata analisi della corrispondenza diplomatica, supportata da varie pièces inedite. Philippe BOUTRY (pp. 39-80) in esemplare lavoro mette in luce il quadro, a volte sofferto, in cui Stendhal vive i suoi rapporti con la Chiesa di Roma, che gli fu sempre profondamente ostile, specie per i suoi scritti, di cui riporta nelle Annexes (I, pp. 69-87 e II, pp. 78-80) giudizi inediti espressi dai prelati romani, relativamente a Rome, Naples et Florence e a Le Rouge et le Noir.

4 Il primo dei due contributi che hanno come oggetto specifico lo Stendhal «archeologo», le cui conclusioni portano ad un sicuro rinnovamento in questo settore fino ad ora troppo poco studiato, è quello di Ludovica CIRRINCIONE D'AMELIo (pp. 81-91) che fornisce una profonda ed esaustiva analisi sul passaggio di Stendhal nel feudo di Canino, di proprietà di Lucien Bonaparte, fratello minore di Napoleone e sugli scavi nella necropoli etrusca, rinvenuta quasi per caso delle terre del principe. Chiude questa prima parte Lucio FELICI (pp. 93-105) che invita a scoprire un cammino che conduca all'effettiva conoscenza dello scrittore grenoblese del grande poeta romano, in un accostamento di opere, in cui par evidente come Stendhal abbia tenuto conto di certi passaggi del Belli.

5 Nella seconda sezione, il primo lavoro è firmato da Laurent STÉFANINI (pp. 109-111) che finemente espone alcuni punti su certi atti del «dilettante Stendhal» console e scrittore, esaminato poi da Giuseppe di GIAcomo (pp. 113-35) in un'accurata rilettura delle sue teorie estetiche, in quanto dalla Storia della pittura in Italia (1817) testimonia come, nell'elaborazione della nozione di «bello ideale moderno» in contrapposizione a quella di «bello ideale antico», Stendhal abbia individuato alcune caratteristiche che saranno proprie del romanzo» (p. 113). Martine REID (pp. 137-151) porta la sua bella analisi su Roma, evidenziando quanto l'Urbe sia «dans l'œuvre de Stendhal une référence incessante, une référence "filée", comme on le dit d'un certain type de métaphore, celle qui imprègne durablement une matière textuelle, en guide le sens, et dicte le foncionnement» (p. 137). Procedendo nell'indagine, viene ben messo in evidenza quanto siano essenziali alla scrittura stendhaliana quelle nuances reattive, quegli incontri con l'arte, con la gente, con la vita stessa di Roma che s'impone sull'opera da Rome, Naples et Florence alle Promenades dans Rome. La città ha dunque un significato mitico che il "flâneur" ha appreso pienamente e che si trasferisce sulla pagina nella sua interezza. Fa parte di questa seconda sezione, un interessante quanto innovativo 
contributo dedicato da Daniela Gallo (pp. 153-69), che pone un Beyle, dichiaratosi lontano dalla scultura, a confronto con Michelangelo e Canova, artisti ammirati e sovente citati nelle sue pagine; per altro «Stendhal - rileva ancora Gallo - sfugge a tutte le possibili etichette nelle sue preferenze in scultura. Michelangelo e Canova restano i suoi idoli, così come immutata resta la sua idiosincrasia contro la scuola berniniana» (p. 168). Lo Stendhal melomane è il soggetto dell'accurata indagine di Ottavio MATTEINI, che molto ha dedicato a quest'aspetto stendhaliano (pp. 171-85). Il critico evidenzia, in un'analisi quanto mai precisa e complessa, l'evoluzione del gusto musicale di Beyle, nel corso dei suoi soggiorni nel nostro paese e nella sua stessa patria: dall'infatuazione per l'opera italiana di un giovane Henri a Novara (o a Ivrea?) per Cimarosa, quindi alla Scala negli anni milanesi, che proseguirà anche in Francia, che comunque in parte si placa durante gli anni consolari a Civitavecchia. Viene quindi condotta un'interessante esame su tale mutamento, con un ricco apparato di citazioni.

6 L'elegante e scrupolosa esegesi di Hélène de JACQUELOT (pp. 187-201) si concentra sulla dimensione di uno Stendhal, ammiratore di Raffaello, investito da una forza della figurazione resa ancor più viva dalla vicinanza del pittore ginevrino Abraham Constantin, riproduttore di genio di Urbino, con cui per alcuni periodi Beyle condivise gli alloggi romani. «Constantin et Raphaël - rileva acutamente la studiosa - occupent donc une place centrale dans cette phase ultérieure d'un apprentissage à la peinture, entre observation et adoration, ce qui explique aussi pourquoi Stendhal devient partie prenante de la rédaction de ce qui deviendra les Idées italiennes» (p. 190), opera firmata da Constantin, nella cui stesura comunque Stendhal ebbe un ruolo fondamentale.

7 Questa sezione viene conclusa dal secondo articolo dedicato alla figura di uno Stendhal archeologo, firmato da Fausto ZEVI e Elena CAGIANO DE AZEVEDo (pp. 203-35), che oltre ad evidenziare la nuova passione stendhaliana per gli scavi, presenta un dettagliato studio delle necropoli etrusche, reperite all'epoca, con ricchezza di particolari e un denso apparato di note. «Stendhal, dunque durante il soggiorno a Civitavecchia, non segue l'archeologia accademica, né quella blasonata dei principi romani, piuttosto è un archeologo per diletto» (p. 234), notano gli autori, sottolineando ancora una volta l'uscita di Beyle da ogni schema prefissato dall'ordine comune, anche nel delicato lavoro di cercatore di tesori.

8 L'ultima sezione sul le scrittore, dal titolo esordisce con un articolo di cui è autore l'insigne critico Gérald RANNAUD (pp. 239-259), tra l'altro curatore della prestigiosa edizione diplomatica de La Vie de Henry Brulard (Paris, Klincksieck, 1996) che conduce con eleganza e precisione il lettore nei percorsi romani di Henri Beyle, caratterizzati da un pregnante lavoro scritturale, dominato tuttavia dall'incompiutezza. "L'atelier romain de Stendhal - nota lo studios - regorge de ces trésors qu'on a méprisés parce qu'on n'y voyait que des échecs. La littérature, au rebours de la peinture, demande le fini, exige un début, et une fin, répugne au fragment» (p. 258). Sandra TERONI propone (pp. 261-76) un articolato studio che si concentra su effettive corrispondenze tra l'opera dedicata all'Urbe e la composizione delle Idées italiennes sur quelques tableaux célèbres, di Abraham Constantin, pubblicate dal Vieusseux nel 1840, che videro tuttavia Stendhal implicato in prima persona nel lavoro dell'amico pittore, un'opera su cui ogni apporto rende sempre più chiara la posizione del grenoblese e meritevole ancora di nuovi approfondimenti. Un nuovo, illuminante studio su Lodovico Ariosto e sulle ripercussioni di quest'ultimo, autore prediletto fin dall'adolescenza, sull'opera di Stendhal, è condotto da Francesco SPANDRI (pp. 277-93) che, utilizzando una 
campionatura di testi, fa emergere, come la ricerca stendhaliana dell'opera rivisitata conduca a un reticolo di circolazioni atte a presupporre nuove funzioni e innovative conclusioni che vengono messe in gioco per concretizzare la storia. In questo l'Ariosto ebbe grande forza. Letizia NORCI CAGIANO (pp. 295-310) si ripropone un interessante studio che vede la stagione romana di Stendhal innestata in La Chartreuse che si svolge in terra lombarda, dove per altro, come ben sostiene Cagiano, «gli agganci colla realtà sussistono e costituiscono il punto di partenza imprescindibile per le costruzioni romanzesche a venire» (p. 304). Conclude il volume un assai rilevante contributo della grande studiosa Béatrice DIDIER (pp. 311-22) dove, in un'allargata investigazione sullo Stendhal «romano» in 1835-1836, viene dimostrato che "[c]'est que Rome lui est indispensable, non seulement à cause de l'activité présente de la Ville et des amitiés qu'il y a nouées, mais parce que Rome est le lieu par excellence de la mémoire» (p. 311). Da segnalare ancora che l'elegante volume è altresì corredato da un ricco apparato iconografico con tracce assai innovative, di profondo interesse per stendhaliani e "amateurs", che lo arricchisce ulteriormente. 